\title{
Advancement in Identification and Classification Framework for Malaria Parasite Based on Image Manipulation
}

\author{
Alamelu. $\mathrm{M}^{* 1}$ and Kavi Priya. C. $\mathrm{U}^{2}$ \\ ${ }^{1}$ Associate Professor, Department of Information Technology, Kumaraguru \\ College of Technology, Coimbatore, India. \\ ${ }^{2} P G$ Scholar, Department of Information Technology, Kumaraguru College \\ of Technology, Coimbatore, India.
}

\section{ABSTRACT}

The transmission of malaria disorder is done by Anopheles genus female mosquitoes. This type of genus mosquitoes is a solitary parasite. The mosquitos that are infected, while sucking the blood it will pass through its salivary glands. The plasmodium then gets injected to the human's blood. In red blood cells the parasites will follow certain division. It gets burst out and it will throw oneself into other RBC's by spreading the parasite. Identifying these type of parasite earlier will reduce the death values across globe. To reduce and identify the parasite in the RBC we proposed an image manipulation - followed totally Malaria disorder discovery framework called the advancement in classification and identification framework for malaria parasite detection. The proposed approach can be analyzed with the data set and categorize the (HSV) histograms, and HSI hue channel histogram using the classification approach and there include the procedure for identification of plasmodium in red blood cells. The Detection and identification of cells based on features algorithm will improve the accuracy of red-blood-cells (RBC) classification using the proposed approach and compare the accuracy with the existing algorithms.

KEY WORDS: ADVANCEMENT IN IDENTIFICATION \& CLASSIFICATION FRAMEWORK, MEAN OUTLINE, PARASITE CELL, RED BLOOD CELLS.

\section{INTRODUCTION}

Irresistible infections result approximately about more than $30 \%$ of deaths globally and basically it emphasized that malaria is one of the three most powerful infections with other harmful inflammation. Agreeing to the later record around 434,000 passing cases had been

\section{ARTICLE INFORMATION}

*Corresponding Author: alamelu.m.it@kct.ac.in

Received 15th Oct 2020 Accepted after revision 10th Dec 2020

Print ISSN: 0974-6455 Online ISSN: 2321-4007 CODEN: BBRCBA

Thomson Reuters ISI Web of Science Clarivate Analytics USA and Crossref Indexed Journal

\section{Clarivate
Analytics}

NAAS Journal Score 2020 (4.31) SJIF: 2020 (7.728)

A Society of Science and Nature Publication,

Bhopal India 2020. All rights reserved.

Online Contents Available at: http//www.bbrc.in/

Doi: http://dx.doi.org/10.21786/bbrc/13.11/7 assessed because of malarial disorder. In the existing method, a conventional microscope is used for malaria parasites. Consumes much time, requires talented labors and the outcome completely depends on the intensive microscopist (Bashar, M. K, 2019). So the detection of stained objects is required for the detection of malaria plasmodium. Moreover, analysation of the stained images for the determination of cells to identify inflamed and noninflamed diagnosis. While detecting the intensity of cells at a fixed and parallel values in image the system can able to detect all the inflamed images (Haixiang, G., Yijing, L., Shang, J., Mingyun, G., Yuanyue, H., \&t Bing, G, 2017). There exist certain techniques for malaria disorder. To classify the malaria parasite certain methods are to be used. The methods include acquisitions of image, preprocessing of image, smoothing of image and extension in image segmentation. There include. 
two phases in architectural model (i) Preparation part and (ii) Identification part. In this work we focus on Advancement in classification and identification framework for malaria disorder. (Priyadarshini Adyasha Pattanaik1, Mohit Mittal, 2019), CAD scheme is used to determine nearness of this disorder in RBC images. To identify a presence of malarial disorder using microscopic images this research presents about CAD scheme using deep learning techniques. The outcome of this work gives good result in detecting the parasite and in exactness.

(Karthik, G., Muttan, S., Saravanan, M. P., Seetharaman, R., \&t Vignesh, V, 2019) provides a novel computerized analysis of malaria via microscopic pictures with the assist of image processing. This community identifies and classifies the crimson blood cells infected by using Plasmodiums like falciparum, vivax, ovale and malariae in skinny blood smears. The image processing set of rules developed is able to locate the infected cells and parasites present in red blood cells. (Bashar, M. K, 2019) examined, a Superintend method to classify malarial disorder stages from microscopy photos has put forward. An analyzation and computational technique has put forward for categorising the lifestyles cycle ranges of the malaria parasite using the some classifiers of support vector machine with numerous features like texture and color. To attain all these benefits, primarily the framework needs to have an excessive computational energy; and furthermore, to make the framework ready, there need to be massive records. There include numerous imperfections which are actually pointed out to distinguish with the tiny investigation.

Related Work: (Yang, F., Poostchi, M., Yu, H., Zhou, Z., Silamut, K., Yu, J., \& Antani, S. 2018), this work deals with the malaria plasmodium by using smartphones. The processing pipeline for computerized parasite detection parasite screening and type. An IGMS is used for immediate screening of a whole thick smear picture a custom designed CNN version then Classifies every candidate as either parasite or historical past. (Mustare, N., \&t Sreelathareddy, V, 2017)it offers by an automatic determination for density of parasite in constant with the microscopic images. This work focuses on a singular approach advanced which can detects all the stages of Plasmodium Vivax and leukocytes. (Khalid, A., Haider, Z., \&t Khosa, I, 2019), this paper recommended a unique technique to discover the existence of today's malarial disorder in blood images. So that the proposed technique has completed considerably better than the opposite methods with a sensitivity latest $97.60 \%$ and specificity present day $95.92 \%$.

(Rollin, G., Lages, J., \&t Shepelyansky, D. L. 2018), this examine, a aggregate technique include dual thresholding and BLOB examination is proposed for categorising the erythrocytes usage of information and also used ARR for computing cellular place. This research, PPV and Sensitivity about $84.43 \%$ and $85.5 \%$ in common is performed. (Roy, K., Sharmin, S., Mukta, R. B. M., Sen, A., Roy, K., Sharmin, S., \& Sen, A. 2018), the usual technique used to come across malaria parasites in blood is a 'gold standard' traditional method. Wherein professionals discover malaria parasites manually by checking each and every slide. The paper suggests the proper dedication of malaria parasites as conventional requirements might also percolate human mistakes. So the method is proposed that the use of image preprocessing,

photograph segmentation, filtering, class and finally the detection of the malaria parasite. (Dave, I. R. 2017), an automated method involving image processing strategies that are effective of finding and acknowledging the parasite infection inside the images viewed through microscope. Finally the proposed technique produce better accuracy for training, and as a final elegance it produce $60 \%$. The set of rules evolved for class in a hierarchical way confirmed exact outcomes. (Mohammed, H. A., \&t Abdelrahman, I. A. M. 2017), in this paper the opportunity of the fast as well as correct computerized analysis of RBC disorders that elaborates a model for detecting and classifying this parasites in sampled blood images received through mild microscope. The BFF neural community produced the very best overall performance.

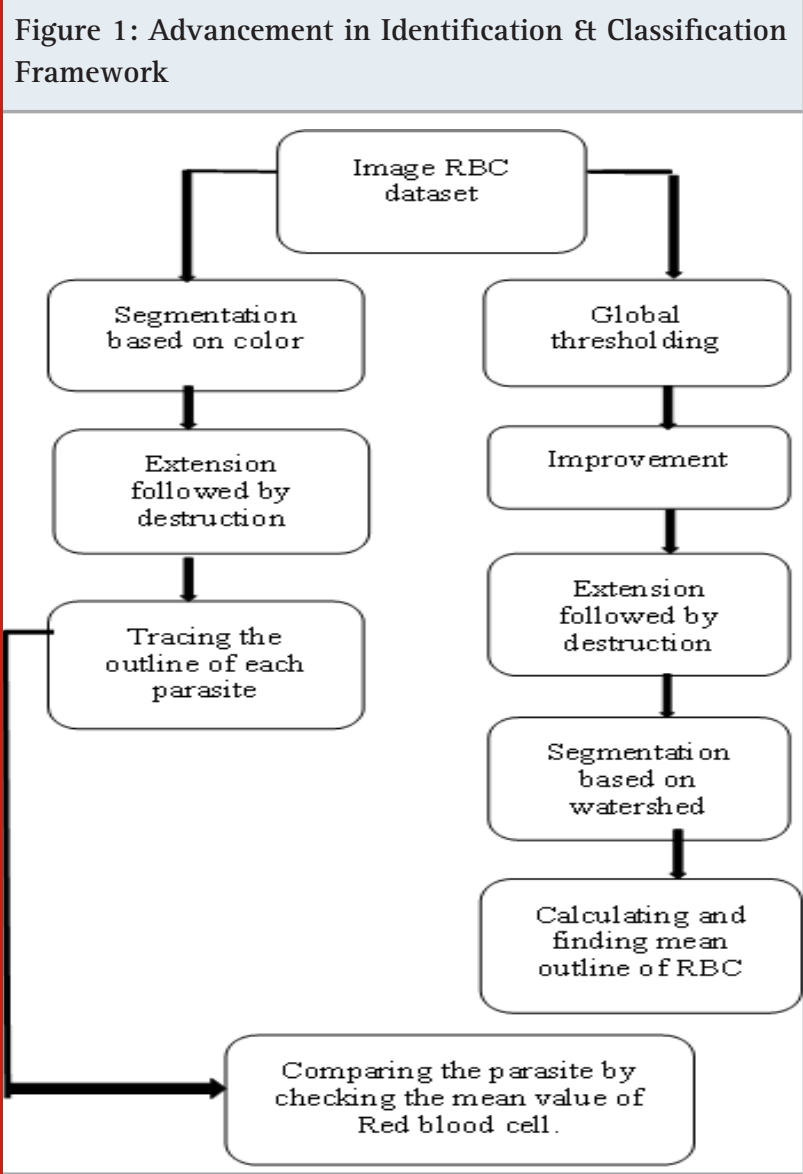

\section{MATERIAL AND METHODS}

Proposed Methodology: The classification framework approach is used to classify and discover malaria parasites as inflamed or uninfected cells. The system 
is extreme to differentiate samples among healthful and parasite infected cells. It deals with the work that contains photo reputation and photo classification that calls for a scientific collection of a couple of activities to achieve the goal. The obtained photos undergo a few pre-processing strategies.

The pre-processing is performed to shape images extra suitable for the subsequent technique. Pre-processing methods contain discount in noise, resizing, and photo differentiation. Normalizing the image size is fundamental for retaining up the spatial resolution of images from exceptional assets. Functions are classified by using the suitable classifier after which it can be set in distinct training. By choosing a parameter suitably which can definitely describe the image Functions are concluded from pix or amounts like signatures and histograms. The procedure utilized for malaria inflamed red blood cells counting is shown in Fig (1).

The steps include,

1. As an input, from the dataset images of red blood cells are taken.

2. Parasite will get segmented based on the color of RBC's.

3. Thresholding is one of best way in segmenting the regions, it will separate the darker and lighter spaces in the cells.

4. The outline of each parasite is traced with that it will calculate the mean outline of RBC.

Red Blood Cell Dataset: The framework chooses the only highlights for making ready by way of disclosing all the separating properties of the approaching picture by means of convolution manner (Bibin, D., Nair, M. S., Et Punitha, P.,2017).. Those highlights then skip via the layers in the network.

Figure 2: Cell image

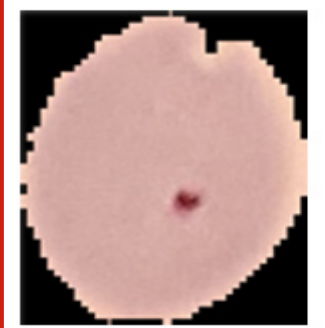

(i) Parasite cell

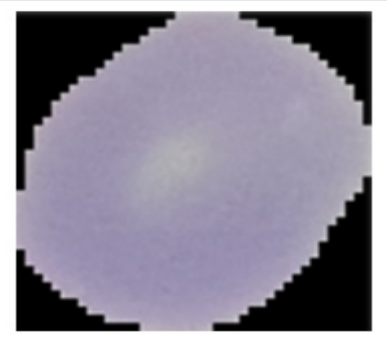

(ii) uninfected cell
Segmentation: An image can be segmented by its essential zone and entity. In Fig 2 images in dataset are named as parasite cell and uninfected cell which shows the difference between them (A. Sai Bharadwaj Reddy and D. Sujitha Juliet, 2019). Exactness of the segmentation explains in the end of successfulness or non-success of procedure analysis. For enhancing the mean value of segmented exactness, a major care must be taken. In many algorithm segmentation similarity, and discontinuity are the general measures of mean values 30
(Roy, K., Sharmin, S., Mukta, R. B. M., Sen, A., Roy, K., Sharmin, S., Ct Sen, A., 2018). Watershed segmentation is a type that has to be used in the case of implementation. In Fig 1 Watershed segmentation is to be used on the red blood cell images to discrete the object that are in collision.

Segmentation Based On Watershed: Segmentation based on watershed is usually used to discrete the objects that are in collision. Thus in this research watershed segmentation is used so can collided red blood cells can be discrete and helpful in separately counting the red blood cells.

Figure 3: Authentic watershed image
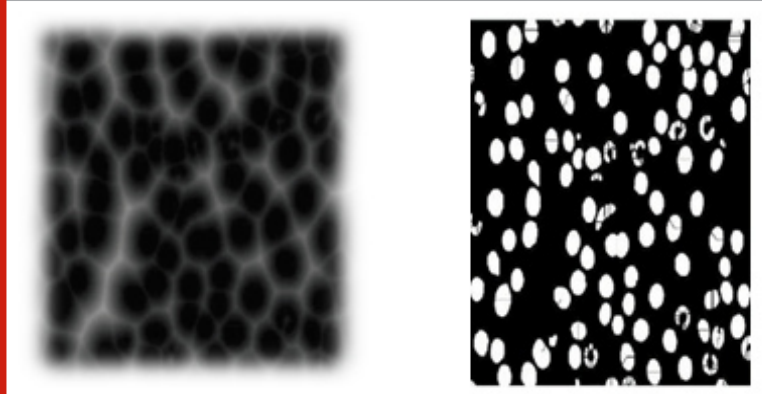

(a) Gradient image

(b) Transformed watershed

1. We assumed two dimensional, gray scaled images as homomorphism for which the parameters of images are given by Fig (3)

2. $a, b$ and the intensity of image is corresponded by the heights.

3. Unwanted noises and some small areas within the image will result generally in local remains catchment basins in lower level.

4. For segmentation authentic image dataset can also be used, but using of gradient photo will usually lead along the edges of object high or low. Thus the ridges of watershed can lie beside the edges of an object. By calculating the spaces between closest non-zeros from each picture element, the distance negation is done.

Thresholding: Exactness of the segmentation explains in the end of successfulness or non-success of procedure analysis. For recognizing the mutual plot region segmentation is used. Thresholding is one of best way in segmenting the regions, it will separate the darker and lighter spaces in the cells. Thresholding value is to 0 for the binary photos created by threshold and 1 to every picture element threshold.

Detection and Identification of Cells: To accomplish the end result by classifying the cell images whether they are inflamed by disorder or not is done using feed forward backpropagation neural network (BFFNN). The generalized property will make a framework to train on a corresponding pair of target values and without even training a framework on every possibly applicable pair 
of input and output. In spite of predicting the number of infected cells, we focused on identifying the infection type.

Figure 4: Perception and recognition of cells based on features

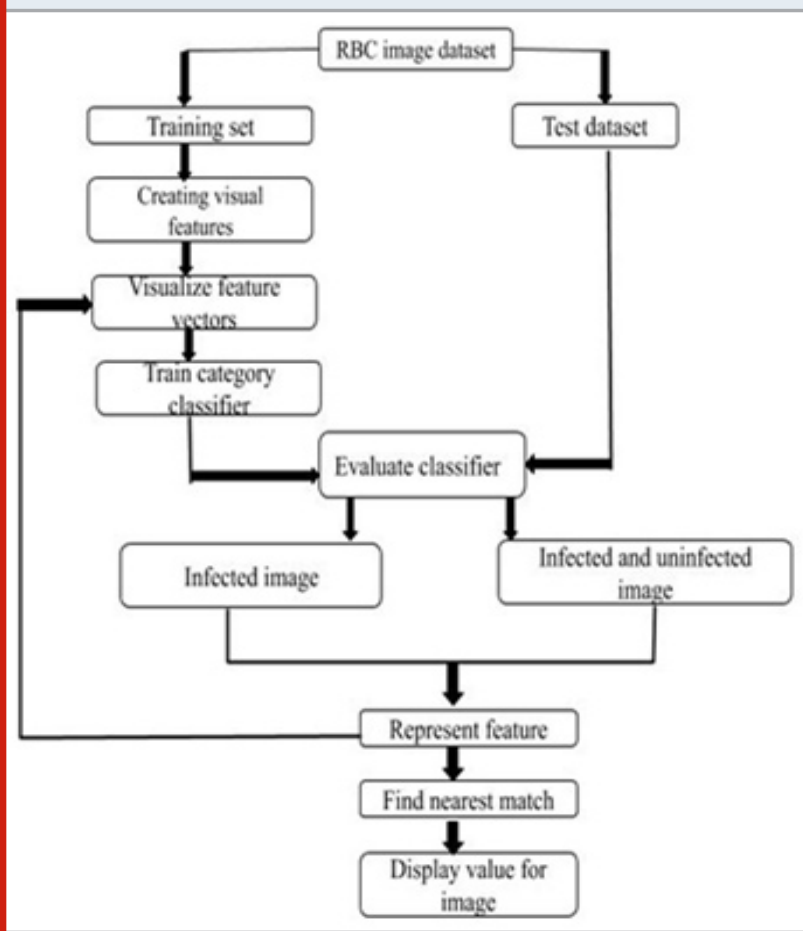

Figure 5: Image database

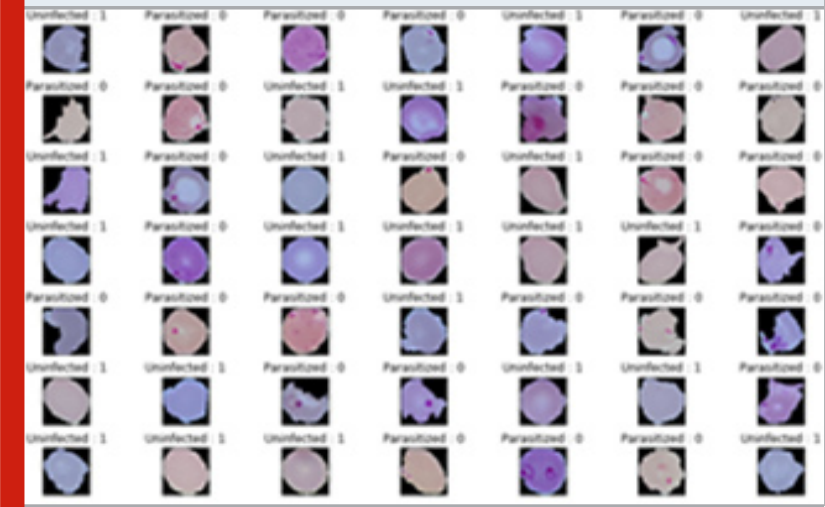

So algorithm will take the input images as example and generate result by differentiating them automatically. The minimization in the squared errors is the goal of training the data and these processes will get stopped, when there is an increase in validation of error. Loaded input parasite images will pass through a neural network and provide results for each and every initialized weight. Back propagation will help in adjusting all the weights in the framework thus the outcome will come nearer to the known.

In Figure 4. Perception and recognition of cells based on features technique follows the process of extracting the features and displaying the estimated value of image.

Table 1. Evaluation of Comparative Approaches

\begin{tabular}{|c|c|c|c|}
\hline YEAR & $\begin{array}{l}\text { EXISTING } \\
\text { RESEARCH } \\
\text { ANALYSIS }\end{array}$ & $\begin{array}{l}\text { TECHNIQUES } \\
\text { USED }\end{array}$ & MERITS \\
\hline 2017 & $\begin{array}{c}\text { Malaria Parasite Detection } \\
\text { from Peripheral Blood Smear } \\
\text { Images using Deep Belief Networks }\end{array}$ & $\begin{array}{l}\text { Deep neural network (DNN), } \\
\text { restricted Boltzmann } \\
\text { machine (RBM) }\end{array}$ & $\begin{array}{l}\text { It helps in advancement of machine } \\
\text { supported based pattern } \\
\text { recognition for malaria plasmodium. }\end{array}$ \\
\hline 2018 & $\begin{array}{c}\text { Malaria Parasite Detection } \\
\text { And Cell Counting For Human } \\
\text { And Mouse Using Thin } \\
\text { Blood Smear Microscopy }\end{array}$ & $\begin{array}{l}\text { Support vector machine, } \\
\text { Artificial neural network, } \\
\text { Multiscale laplacian of } \\
\text { gaussian cell detection method }\end{array}$ & $\begin{array}{l}\text { Approximate centers for separate } \\
\text { cells are detected and it provides } \\
\text { cell detection of accuracy in higher } \\
\text { level as well as speed processing. }\end{array}$ \\
\hline 2018 & $\begin{array}{l}\text { Image analysis and machine } \\
\text { learning for detecting malaria. }\end{array}$ & $\begin{array}{c}\text { Gray Level Run Length Matrix, } \\
\text { Quantitative PhaseImaging, SUSAN, } \\
\text { Support Vector Machine }\end{array}$ & $\begin{array}{c}\text { It suits malaria } \\
\text { patients' test level in larger } \\
\text { and allows more standardized } \\
\text { corrections, value testing in } \\
\text { extensive levels. }\end{array}$ \\
\hline 2020 & $\begin{array}{l}\text { Identification of Plasmodium } \\
\text { falciparum Stages Using } \\
\text { Support Vector Machine Method }\end{array}$ & $\begin{array}{l}\text { Gray Level Co-occurrence } \\
\text { Matrix , Otsu threshold method for } \\
\text { segmentation }\end{array}$ & $\begin{array}{l}\text { It produced an accuracy of } \\
\text { nearly 92\% using extracted } \\
\text { features combined with the } \\
\text { characteristics of morphology. }\end{array}$ \\
\hline
\end{tabular}

1. Firstly the specifications are compared with the estimated values of the red blood cell images to check inflamed and non-inflamed cells.

2. But if the value of the cell are equal or less than mean value, it will not do anything.

3. While comparing, when the outside of plasmodium is higher than the mean value of red blood cells, it will highlight it with a circle on all sides of plasmodium 
and inflamed cells are get counted.

4. Cells are highlighted by circles if the value of parasites is higher than mean value.

5. If outside of plasmodium lies on or lower than estimated value of the red blood cells it won't do anything.

\section{RESULTS AND DISCUSSION}

The dataset taken into consider are of images that are split into train and test data which contain both infected and uninfected cells of nearly 28,000 images. Afterward the Convolution neural network appears is prepared, and the appearance are anticipated. These yields helps in choosing the contaminated as well as sound sampled blood. To maintain uniformity on the images within the dataset pre-processing is required. It makes a difference in preparing the show far off superior by giving more varieties in pictures and by centering on the specified parameters. Reduction of images are done by annotating the images, where the falsely and suspicious images are removed. In Figure. 2 datasets are mentioned as infected (0) cells and uninfected (1) cells. The number of falsely and suspicious images are about 648 removing that from dataset results in 26,164 images. To predict the output these labels are to be used in the model.

Table 1. represents the evaluation of the comparative analysis of the proposed approach with the existing approaches. The proposed approach used an authenticated red blood cell images among nearly 2000 cell images. Identification of malaria disorder is done by using image manipulation, segmentation based on watershed, extension followed by destruction. The testing analysis is done by comparing the separated perimeter cell.

\section{CONCLUSION}

In the proposed Advancement in Identification $\mathrm{Ct}$ Classification Framework approach we analyzed the identification of malaria parasites based on image manipulation techniques using few deep learning mechanisms. It will focus on detection of infected red blood cells based on its features. By using this proposed approach both the infected and uninfected red blood cells will obtain better accuracy in comparison with the existing systems. In spite of predicting the number of infected cells, we focused on identifying the infection type. So algorithm will take the input images as example and generate result by differentiating them automatically. The approach can be further expanded by deploying certain techniques and measures in detecting this malaria disorder in earliest.

\section{REFERENCES}

Abbas, N., Saba, T., Rehman, A., Mehmood, Z., Kolivand, H., Uddin, M., \&t Anjum, A. (2019). Plasmodium life cycle stage classification based quantification of malaria parasitaemia in thin blood smears. Microscopy research and technique,IEEE Access, 82(3), 283-295.

Bashar, M. K. (2019, November)” Improved Classification of Malaria Parasite Stages with Support Vector Machine Using Combined Color and Texture Features". In 2019 IEEE Healthcare Innovations and Point of Care Technologies,(HI-POCT) (pp. 135-138). IEEE.

Bibin, D., Nair, M. S., \&t Punitha, P. (2017). Malaria parasite detection from peripheral blood smear images using deep belief networks. IEEE Access, 5, 90999108.

Dave, I. R. (2017, March). Image analysis for malaria parasite detection from microscopic images of thick blood smear. In 2017 International Conference on Wireless Communications, Signal Processing and Networking (WiSPNET) (pp. 1303-1307). IEEE.

Ghanmode, B. D., \&t Paikrao, P. L. (2018, October). "Malaria Parasite Concentration Determination Using Digital Image Processing” In 2018 4th International Conference for Convergence in Technology (I2CT) (pp. 1-4). IEEE.

Gonzalez, R. C., Woods, R. E., \&t Eddins, S. L. (2004). Digital image processing using MATLAB. Pearson Education India.

Haixiang, G., Yijing, L., Shang, J., Mingyun, G., Yuanyue, H., \& Bing, G. (2017). Learning from classimbalanced data: Review of methods and applications. Expert Systems with Applications, IEEE Access,vol.73, 220-239.

Hu, C., Ju, R., Shen, Y., Zhou, P., \&t Li, Q. (2016, May). Clinical decision support for alzheimer's disease based on deep learning and brain networks. In 2016 IEEE International Conference on Communications (ICC) (pp. 1-6). IEEE.

Karthik, G., Muttan, S., Saravanan, M. P., Seetharaman, R., \& Vignesh, V. (2019, January). "Automated Malaria Diagnosis Using Microscopic Images”. In 2019 Third International Conference on Inventive Systems and Control (ICISC) (pp. 514-517). IEEE.

Khalid, A., Haider, Z., \& Khosa, I. (2019, January). Malarial Parasite Detection and Recognition using Microscopic Images. In 2019 16th International Bhurban Conference on Applied Sciences and Technology (IBCAST) (pp. 304-308). IEEE.

Mohammed, H. A., \&t Abdelrahman, I. A. M. (2017, January). Detection and classification of malaria in thin blood slide images. In 2017 International Conference on Communication, Control, Computing and Electronics Engineering (ICCCCEE) (pp. 1-5). IEEE.

Mustare, N., \&t Sreelathareddy, V. (2017, September). :Development of automatic identification and classification system for malaria parasite in thin blood smears based on morphological techniques". In 2017 IEEE International Conference on Power, Control, Signals and Instrumentation Engineering (ICPCSI) (pp. 3006-3011). IEEE. 
Poostchi, M., Ersoy, I., McMenamin, K., Gordon, E., Palaniappan, N., Pierce, S., \&t Palaniappan, K. (2018). Malaria parasite detection and cell counting for human and mouse using thin blood smear microscopy. Journal of Medical Imaging,IEEE Access, 5(4), 044506.

Poostchi, M., Silamut, K., Maude, R. J., Jaeger, S., \& Thoma, G. (2018). Image analysis and machine learning for detecting malaria. Translational Research, IEEE Access, 194, 36-55.

Rajaraman, S., Antani, S. K., Poostchi, M., Silamut, K., Hossain, M. A., Maude, R. J., \&t Thoma, G. R. (2018). Pre-trained convolutional neural networks feature extractors toward improved malaria parasite detection in thin blood smear images. IEEE Access,vol. 6, 4568. Ravi, D., Wong, C., Deligianni, F., Berthelot, M., AndreuPerez, J., Lo, B., \&t Yang, G. Z. (2016). Deep learning for health informatics. IEEE journal of biomedical and health informatics, IEEE Access,21(1), 4-21.

Rollin, G., Lages, J., \&t Shepelyansky, D. L. (2019).
World Influence of Infectious Diseases From Wikipedia Network Analysis. IEEE Access, 7, 26073-26087.

Roy, K., Sharmin, S., Mukta, R. B. M., Sen, A., Roy, K., Sharmin, S., \& Sen, A. (2018). Detection of malaria parasite in giemsa blood sample using image processing. International Journal of Computer Science \& Information Technology (Ijcsit), IEEE.

Setianingrum, A. H., Wardhani, L. K., Ridwan, A. F., Et Nasution, S. F. (2019, November). Identification of Plasmodium falciparum Stages Using Support Vector Machine Method. In 2019 7th International Conference on Cyber and IT Service Management (CITSM) (Vol. 7, pp. 1-5). IEEE.

Yang, F., Poostchi, M., Yu, H., Zhou, Z., Silamut, K., Yu, J., \&t Antani, S. (2019). "Deep learning for smartphonebased malaria parasite detection in thick blood smears". IEEE Journal of Biomedical and Health Informatics, 24(5), 1427-1438. 